\title{
Fluorescence Microscopy Datasets for Training Deep Neural Networks
}

\section{Guy M. Hagen ${ }^{1}$, Justin Bendesky¹, Rosa Machado ${ }^{1}$, Tram-Anh Nguyen², Tanmay Kumar ${ }^{3}$, Jonathan Ventura ${ }^{3}$}

\author{
${ }^{1}$ UCCS BioFrontiers Center, University of Colorado at Colorado Springs, 1420 Austin Bluffs Parkway, \\ Colorado Springs, Colorado, 80918. \\ ${ }^{2}$ George Mason University, 4400 University Drive, Fairfax, Virginia, 22030. \\ ${ }^{3}$ Department of Computer Science and Software Engineering, California Polytechnic State University, \\ San Luis Obispo, California, 93407
}

\begin{abstract}
Background: Fluorescence microscopy is an important technique in many areas of biological research. Two factors which limit the usefulness and performance of fluorescence microscopy are photobleaching of fluorescent probes during imaging, and, when imaging live cells, phototoxicity caused by light exposure. Recently developed methods in machine learning are able to greatly improve the signal to noise ratio of acquired images. This allows researchers to record images with much shorter exposure times, which in turn minimizes photobleaching and phototoxicity by reducing the dose of light reaching the sample.
\end{abstract}

Findings: To employ deep learning methods, a large amount of data is needed to train the underlying convolutional neural network. One way to do this involves use of pairs of fluorescence microscopy images acquired with long and short exposure times. We provide high quality data sets which can be used to train and evaluate deep learning methods under development.

Conclusion: The availability of high quality data is vital for training convolutional neural networks which are used in current machine learning approaches.

Keywords: fluorescence microscopy, deep learning, convolutional neural networks

\section{Data description}

\section{Context}

Fluorescence microscopy is an important technique in many areas of biomedical research, but its use can be limited by photobleaching of fluorescent probe molecules caused by the excitation light which is used. In addition, reactive oxygen species which are generated by exposing samples to light can cause cell damage and even cell death, limiting imaging of live cells [1,2]. Many strategies have been devised to overcome this problem including the use of specialized culture media [3,4], pulsed excitation [5], or more elaborate methods such as controlled light exposure microscopy $[6,7]$.

Another approach involves recording of fluorescence microscopy images with short exposure times, low excitation light intensity, or both. This results in images with low signal to noise ratios (SNRs), which can subsequently be improved using a variety of image restoration approaches [8-12]. Noise in low light images of this type typically follows a Poisson-Gaussian distribution. This condition makes solving the inverse problem which arises in image restoration methods very difficult, leading to a variety of approximate methods [13].

Recently, deep learning methods in artificial intelligence [14] have been applied to many problems in image analysis, including those in optical microscopy [15-18] and in image denoising [19,20]. Deep learning approaches typically require a large amount of data to train the underlying convolutional neural network [21], however, such datasets are not always available. Here we provide fluorescence microscopy datasets which can be used to train and evaluate neural networks for the purpose of image denoising. The 
dataset consists of pairs of images acquired with different exposure times. After training, the network can subsequently be used to enhance the SNR of newly acquired images.

One advantage of deep learning methods is that they can learn a task such as denoising from the data itself, thus providing a sample-specific method which does not depend on a physical model. Once a network has been trained, subsequent image denoising using a convolutional neural network is fast compared to traditional methods which are typically much slower.

In addition to providing the datasets, we evaluated the performance of a recently proposed neural network for content-aware image restoration (CARE) of fluorescence microscopy images [17]. To do this we used CSBDeep [22], a toolbox for implementation of the CARE network. This network uses a series of convolutional layers in a U-Net architecture [23].

We also evaluated a self-supervised learning approach called a blindspot neural network [24], an extension of the Noise2Void approach [25]. This method learns denoising using only the noisy data. It uses a U-Net style architecture but uses careful padding and cropping to force the network to learn to predict the value of each denoised pixel based on the neighborhood of that pixel in the noisy input. We used our own implementation in Python using the Keras library.

\section{Methods}

\section{Fluorescence Microscopy}

We acquired datasets 1 - 3 using an IX83 microscope equipped with UplanSApo 60×/1.3 NA oil immersion and UplanSApo 20×/0.75 NA air objectives (Olympus, Tokyo, Japan), Zyla 4.2-plus sCMOS camera (Andor, Belfast, UK), and SpectraX light source (Lumencor, Beaverton, OR, USA). Focusing was achieved using a piezo-Z stage (Applied Scientific Instrumentation, Eugene, OR, USA). The system was controlled by IQ3 software (Andor). We used fluorescence filter set 59022 (Chroma, Bellows Falls, VT, USA). Dataset 4 was acquired with a SP5 laser scanning confocal microscope (Leica, Mannheim, Germany) using $488 \mathrm{~nm}$ and $543 \mathrm{~nm}$ lasers and a HCX PL APO CS 63×/1.4 NA oil immersion objective (Leica).

The sample in all cases was a FluoCells \#1 prepared slide (Molecular Probes, Eugene, OR, USA). This slide contains bovine pulmonary artery endothelial cells stained with MitoTracker Red CMXRos (labels mitochondria) and AlexaFluor 488 phalloidin (labels actin).

\section{Data Analysis}

Each dataset consisted of images of size $2048 \times 2048$ pixels, where the last ten percent were used for testing and the remaining were used for training. In datasets 1, 2, and 3 we had 100 image pairs in each dataset and in dataset 4 (confocal), we had 79 image pairs and the image size was $1024 \times 1024$ pixels.

To train the CARE network, we used the following configuration. We used the ADAM optimizer [26], the training batch size was 16 images, the number of training epochs was 200 , the initial learning rate was 0.0004, and the iterations per epoch (training steps) was 400. In sampling the training images, 800 patches per image of size 64 pixels by 64 pixels were used to train the CARE network. In all experiments, images were split according to the ratio 5:1 for training and validation respectively.

Following the standard implementation of the CSBDeep network, we used the Laplacian loss function

$$
L_{\text {Laplace }}(\theta)=\frac{1}{T} \frac{1}{N} \sum_{t=1}^{T} \sum_{i=1}^{N} \frac{\left|y_{i}^{t}-\mu_{\theta}\left(x^{t}\right)_{i}\right|}{\sigma_{\theta}\left(x^{t}\right)_{i}}+\log \sigma_{\theta}\left(x^{t}\right)_{i}
$$

where $\mathrm{T}$ is the number of training images, $\mathrm{N}$ is the number of pixels per image, $y^{t}$ is the ground truth pixel value, $x^{t}$ is the input pixel, $\mu$ and $\sigma$ are the mean and variance of the predicted pixel distribution. 
To train the blindspot network, we used the same configuration described in Laine, et al (2019). We used the ADAM optimizer and trained on random crops of size $128 \times 128$ pixels using a mean-squared-error loss function. We trained over 200 epochs with 400 steps per epoch and an initial learning rate of 0.0003 . In all experiments, the last five images of the training set were withheld for validation. For comparison we used a standard denoising method, block matching and 3D filtering (BM3D) [27].

\section{Results}

We acquired four datasets under different conditions. Table 1 provides an overview of the four datasets. In the widefield data, we used the Lumencor light source control software to adjust the illumination intensity such that the desired signal to noise levels were achieved.

Table 1: Overview of the datasets

\begin{tabular}{|c|c|c|c|c|}
\hline data set & $1(60 X$ noise level 1$)$ & $2(60 X$ noise level 2) & $3(20 X)$ & 4 (confocal) \\
\hline microscope & Widefield & Widefield & Widefield & Confocal \\
\hline objective & $60 \times / 1.35 \mathrm{NA}$ oil immersion & $60 \times / 1.35 \mathrm{NA}$ oil immersion & $20 \times / 0.75 \mathrm{NA}$ air & $63 \times / 1.4 \mathrm{NA}$ oil immersion \\
\hline pixel size & $108 \mathrm{~nm}$ & $108 \mathrm{~nm}$ & $325 \mathrm{~nm}$ & $96 \mathrm{~nm}$ \\
\hline \multirow[t]{4}{*}{ Exposure times } & high exposure (actin): $400 \mathrm{~ms}$ & high exposure (actin): $1000 \mathrm{~ms}$ & high exposure (actin): $500 \mathrm{~ms}$ & 0.7 images/sec \\
\hline & low exposure (actin): $20 \mathrm{~ms}$ & low exposure (actin): $15 \mathrm{~ms}$ & low exposure (actin): $20 \mathrm{~ms}$ & 0.7 images/sec \\
\hline & $\begin{array}{c}\text { high exposure } \\
\text { (mitochondria): } 400 \mathrm{~ms}\end{array}$ & $\begin{array}{c}\text { high exposure } \\
\text { (mitochondria): } 600 \mathrm{~ms}\end{array}$ & $\begin{array}{c}\text { high exposure } \\
\text { (mitochondria): } 400 \mathrm{~ms}\end{array}$ & 0.7 images/sec \\
\hline & $\begin{array}{c}\text { low exposure } \\
\text { (mitochondria): } 20 \mathrm{~ms}\end{array}$ & $\begin{array}{c}\text { low exposure } \\
\text { (mitochondria): } 10 \mathrm{~ms}\end{array}$ & $\begin{array}{c}\text { low exposure } \\
\text { (mitochondria): } 15 \mathrm{~ms}\end{array}$ & 0.7 images/sec \\
\hline image size, pixels & $2048 \times 2048$ & $2048 \times 2048$ & $2048 \times 2048$ & $1024 \times 1024$ \\
\hline
\end{tabular}

After data acquisition, we tested three different methods for image denoising. Figure 1 shows the original low exposure image (raw), the matching high exposure image (ground truth), and the results of the CARE method, the blindspot method, and a standard denoising method (BM3D). For this comparison we selected an image pair from data set 1 (60X noise level 1). 
bioRxiv preprint doi: https://doi.org/10.1101/2020.06.17.158097; this version posted June 18, 2020. The copyright holder for this preprint (which was not certified by peer review) is the author/funder, who has granted bioRxiv a license to display the preprint in perpetuity. It is made available under aCC-BY-NC-ND 4.0 International license.

actin
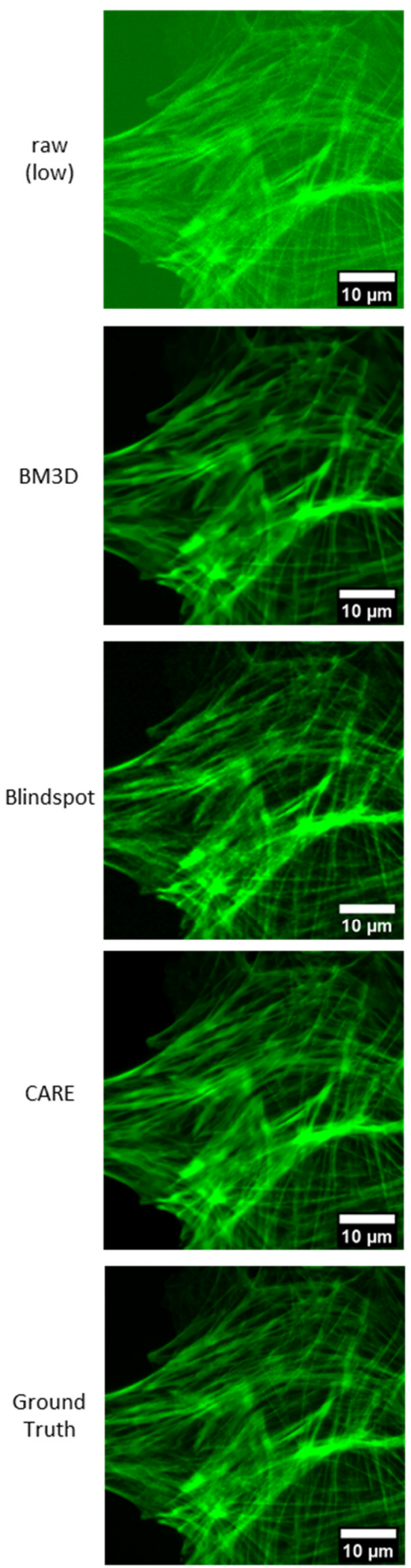

mitochondria
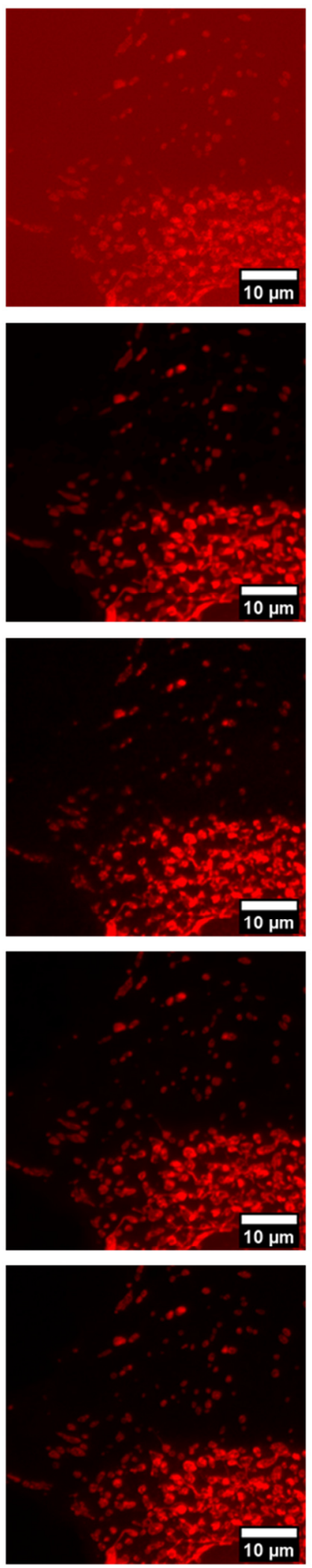

merge
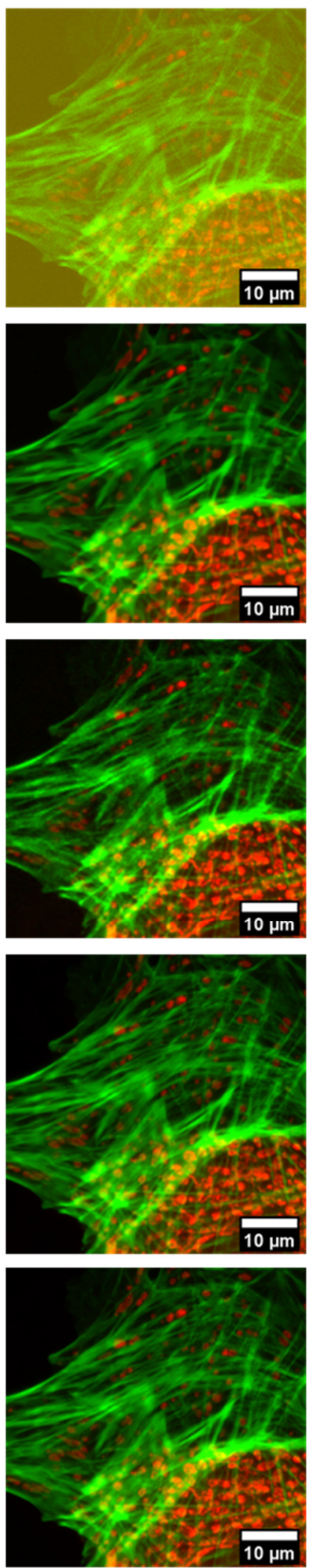

Figure 1: Results of denoising methods. Shown are selected images from dataset 1 (60X noise 1). 
Table 2 provides average metrics for the denoising performance for each method on each dataset. We used two metrics: peak signal-to-noise ratio (PSNR) and structural similarity (SSIM). We normalized both images by clipping values below the $1^{\text {st }}$ percentile and above the $99^{\text {th }}$ percentile. We then scaled and shifted both images to minimize the mean squared error (MSE) between them [17].

$$
M S E=\frac{1}{m n} \sum_{i=0}^{m-1} \sum_{j=}^{n-1}[I(i, j)-K(i, j)]^{2}
$$

Where $I$ is a high SNR image and $K$ is the corresponding low SNR image after restoration.

Finally, the PSNR metric was calculated as

$$
P S N R=10 \log _{10}\left(\frac{1}{M S E}\right)
$$

The SSIM metric [28] is an image quality metric designed to approximate human perception of similarity to a reference image. Unlike PSNR, the metric takes into account structural information in the image. The SSIM metric ranges from 0 to 1 with a greater number indicating higher quality.

As shown in Table 2, the unsupervised blindspot method is the weakest performer on both metrics. BM3D is better on both metrics but surpassed by the supervised CARE method on almost all datasets. All methods exhibit an approximately $10 \mathrm{~dB}$ drop in PSNR or greater on the noisier datasets (Noise 2) in comparison to Noise 1. Each method also performed about 6-7 dB worse on $20 \times$ magnification data in comparison to the $60 \times$ magnification data.

Visual inspection of the restored images (example shown in Figure 1) shows that, despite having high SSIM scores, the BM3D tends to blur the images more than the other methods. The results of the blindspot method are noticeably noisier than the results of the other methods.

Table 2. Average PSNR and SSIM results $(n=10)$

\begin{tabular}{|c|c|c|c|c|c|c|c|c|}
\hline & \multicolumn{4}{|c|}{ PSNR, dB } & \multicolumn{4}{c|}{ SSIM } \\
\hline Dataset & $\begin{array}{c}\text { Raw } \\
\text { (low) }\end{array}$ & CARE & Blindspot & BM3D & Original & CARE & Blindspot & BM3D \\
\hline Actin 20x & 24.10 & 31.15 & 28.98 & 30.31 & 0.366 & 0.87 & 0.81 & 0.86 \\
\hline Actin 60x (noise 1) & 27.95 & 38.43 & 33.06 & 36.09 & 0.60 & 0.95 & 0.88 & 0.92 \\
\hline Actin 60x (noise 2) & 18.34 & 24.90 & 23.73 & 25.12 & 0.09 & 0.47 & 0.31 & 0.51 \\
\hline Mito 20x & 24.41 & 32.13 & 28.37 & 29.50 & 0.33 & 0.88 & 0.73 & 0.81 \\
\hline Mito 60x (noise 1) & 27.91 & 39.03 & 34.22 & 36.31 & 0.55 & 0.96 & 0.90 & 0.93 \\
\hline Mito 60x (noise 2) & 19.95 & 26.15 & 23.21 & 24.59 & 0.13 & 0.63 & 0.25 & 0.41 \\
\hline Actin Confocal & 24.65 & 27.31 & 26.66 & 27.16 & 0.67 & 0.76 & 0.75 & 0.78 \\
\hline Mito Confocal & 22.07 & 27.31 & 24.75 & 26.91 & 0.52 & 0.62 & 0.76 & 0.74 \\
\hline
\end{tabular}

Table 3 presents an comparison of the methods in terms of computation time. Using a single Nvidia V100 GPU, the CARE network took about 35 minutes to train on a single dataset while the blindspot network took about 2.6 hours. The CARE network took about 1 second to process a single image while the blindspot network took over 3 seconds. The BM3D method does not require training but took about 50 seconds to process a single image in MATLAB on a $2.6 \mathrm{GHz}$ Intel Core i3-7100U processor.

Table 3. Training and processing times

\begin{tabular}{|c|c|c|}
\hline & Training time, sec & Processing time for 1 image, sec \\
\hline CARE & 2143.01 & 0.90 \\
\hline Blindspot & 9450.86 & 3.35 \\
\hline BM3D & - & 50 \\
\hline
\end{tabular}




\section{Reuse potential}

The provided data can be used to implement new methods in machine learning or to test modifications of existing approaches. The data can be used to evaluate methods for denoising, super-resolution, or generative modeling, as well as new image quality metrics, for example. The data could also be used to evaluate the generalization ability of methods trained on one type of data and tested on another. High quality, publicly available data of this type has been lacking.

\section{Availability of supporting data}

All raw and analyzed data is available on GigaDB at http://gigadb.org/site/index. All files and data are distributed under the Creative Commons $\mathrm{CC} 0$ waiver, with a request for attribution. The data are organized into 8 main folders for the 4 different data sets (see Table 4).

Table 4. Description of the data files

\begin{tabular}{|c|c|c|}
\hline Folder & Files & File Size \\
\hline & actin-60x-noise1-highsnr.tif & $838.9 \mathrm{MB}$ \\
\hline 01-actin-60x-noise1 & actin-60x-noise1-lowsnr.tif & $838.9 \mathrm{MB}$ \\
\hline 01-mito-60x-noise1 & mito-60x-noise1-highsnr.tif & $838.9 \mathrm{MB}$ \\
\hline & mito-60x-noise1-lowsnr.tif & $838.9 \mathrm{MB}$ \\
\hline 02-actin-60x-noise2 & actin-60x-noise2-highsnr.tif & $838.9 \mathrm{MB}$ \\
\hline & actin-60x-noise2-lowsnr.tif & $838.9 \mathrm{MB}$ \\
\hline 02-mito-60x-noise2 & mito-60x-noise2-highsnr.tif & $838.9 \mathrm{MB}$ \\
\hline 03-actin-confocal & mito-60x-noise2-lowsnr.tif & $165.7 \mathrm{MB}$ \\
\hline 03-mito-confocal & actin-confocal-highsnr.tif & $165.7 \mathrm{MB}$ \\
\hline & actin-confocal-lowsnr.tif & $165.7 \mathrm{MB}$ \\
\hline & mito-confocal-highsnr.tif & $165.7 \mathrm{MB}$ \\
\hline & mito-confocal-lowsnr.tif & $838.9 \mathrm{MB}$ \\
\hline & actin-20x-noise1-highsnr.tif & $838.9 \mathrm{MB}$ \\
\hline
\end{tabular}

\section{Abbreviations}

SSIM: structural similarity index

PSNR: peak signal to noise ratio

NA: numerical aperture

\section{Ethics approval and consent to participate}

Not applicable

\section{Consent for publication}

Not applicable

\section{Competing interests}

The authors declare that they have no competing interests.

\section{Funding}


This work was supported by the National Institutes of Health grant number 1R15GM128166-01. This work was also supported by the UCCS BioFrontiers center. The funding sources had no involvement in study design; in the collection, analysis and interpretation of data; in the writing of the report; or in the decision to submit the article for publication. This material is based in part upon work supported by the National Science Foundation under Grant Number 1727033. Any opinions, findings, and conclusions or recommendations expressed in this material are those of the authors and do not necessarily reflect the views of the National Science Foundation.

\section{Author Contributions}

TN: analyzed data

JB: acquired data

RM: acquired data

TK: analyzed data

$\mathrm{JV}$ : conceived project, analyzed data, supervised research, wrote the paper

GH: conceived project, acquired data, analyzed data, supervised research, wrote the paper

\section{References}

1. J. Icha, M. Weber, J. C. Waters, and C. Norden, "Phototoxicity in live fluorescence microscopy, and how to avoid it," BioEssays 39, 1700003 (2017).

2. R. Dixit and R. Cyr, "Cell damage and reactive oxygen species production induced by fluorescence microscopy: effect on mitosis and guidelines for non-invasive fluorescence microscopy," Plant J. 36, 280-290 (2003).

3. A. M. Bogdanov, E. A. Bogdanova, D. M. Chudakov, T. V Gorodnicheva, S. Lukyanov, and K. A. Lukyanov, "Cell culture medium affects GFP photostability: A solution," Nat. Methods 6, 859860 (2009).

4. A. M. Bogdanov, E. I. Kudryavtseva, and K. A. Lukyanov, "Anti-Fading Media for Live Cell GFP Imaging," PLoS One 7, e53004 (2012).

5. T. Nishigaki, C. D. Wood, K. Shiba, S. A. Baba, and A. Darszon, "Stroboscopic illumination using light-emitting diodes reduces phototoxicity in fluorescence cell imaging," Biotechniques 41, 191197 (2006).

6. R. A. Hoebe, C. H. Van Oven, T. W. J. Gadella, P. B. Dhonukshe, C. J. F. Van Noorden, and E. M. M. Manders, "Controlled light-exposure microscopy reduces photobleaching and phototoxicity in fluorescence live-cell imaging," Nat. Biotechnolgy 25, 249-253 (2007).

7. W. Caarls, B. Rieger, A. H. B. De Vries, D. J. Arndt-Jovin, and T. M. Jovin, "Minimizing light exposure with the programmable array microscope," J. Microsc. 101-110 (2010).

8. M. Arigovindan, J. C. Fung, D. Elnatan, V. Mennella, Y.-H. M. Chan, M. Pollard, E. Branlund, J. W. Sedat, and D. A. Agard, "High-resolution restoration of 3D structures from widefield images with extreme low signal-to-noise-ratio," Proc. Natl. Acad. Sci. 110, 17344-17349 (2013).

9. J. B. Sibarita, "Deconvolution microscopy," in Advances in Biochemical Engineering/Biotechnology (Springer, Berlin, Heidelberg, 2005), Vol. 95, pp. 201-243.

10. J. Boulanger, C. Kervrann, P. Bouthemy, P. Elbau, J.-B. Sibarita, and J. Salamero, "Patch-based 
nonlocal functional for denoising fluorescence microscopy image sequences.," IEEE Trans. Med. Imaging 29, 442-54 (2010).

11. E. Soubies, F. Soulez, M. T. McCann, T. Pham, L. Donati, T. Debarre, D. Sage, and M. Unser, "Pocket guide to solve inverse problems with GlobalBioIm," Inverse Probl. 35, 104006 (2019).

12. P. J. Verveer, M. J. Gemkow, and T. M. Jovin, "A comparison of image restoration approaches applied to three-dimensional confocal and wide-field fluorescence microscopy," J. Microsc. 193, 50-61 (1999).

13. S. Setzer, G. Steidl, and T. Teuber, "Deblurring Poissonian images by split Bregman techniques," J. Vis. Commun. Image Represent. 21, 193-199 (2010).

14. Y. Lecun, Y. Bengio, and G. Hinton, "Deep learning," Nature 521, 436-444 (2015).

15. W. Ouyang, A. Aristov, M. Lelek, X. Hao, and C. Zimmer, "Deep learning massively accelerates super-resolution localization microscopy," Nat. Biotechnol. 36, 460-468 (2018).

16. Y. Rivenson, Z. Göröcs, H. Günaydin, Y. Zhang, H. Wang, and A. Ozcan, "Deep learning microscopy," Optica 4, 1437 (2017).

17. M. Weigert, U. Schmidt, T. Boothe, A. Müller, A. Dibrov, A. Jain, B. Wilhelm, D. Schmidt, C. Broaddus, S. Culley, M. Rocha-Martins, F. Segovia-Miranda, C. Norden, R. Henriques, M. Zerial, M. Solimena, J. Rink, P. Tomancak, L. Royer, F. Jug, and E. W. Myers, "Content-aware image restoration: pushing the limits of fluorescence microscopy," Nat. Methods 15, 1090-1097 (2018).

18. E. Nehme, L. E. Weiss, T. Michaeli, and Y. Shechtman, "Deep-STORM: super-resolution singlemolecule microscopy by deep learning," Optica 5, 458 (2018).

19. K. Zhang, W. Zuo, Y. Chen, D. Meng, and L. Zhang, "Beyond a Gaussian Denoiser: Residual Learning of Deep CNN for Image Denoising," IEEE Trans. Image Process. 26, 3142-3155 (2017).

20. X.-J. Mao, C. Shen, and Y.-B. Yang, "Image Restoration Using Convolutional Auto-encoders with Symmetric Skip Connections," (2016).

21. D. E. Rumelhart, G. E. Hinton, and R. J. Williams, "Learning representations by back-propagating errors," Nature 323, 533-536 (1986).

22. "CSBDeep," https://csbdeep.bioimagecomputing.com/. Accessed June 4, 2020.

23. T. Falk, D. Mai, R. Bensch, Ö. Çiçek, A. Abdulkadir, Y. Marrakchi, A. Böhm, J. Deubner, Z. Jäckel, K. Seiwald, A. Dovzhenko, O. Tietz, C. Dal Bosco, S. Walsh, D. Saltukoglu, T. L. Tay, M. Prinz, K. Palme, M. Simons, I. Diester, T. Brox, and O. Ronneberger, "U-Net: deep learning for cell counting, detection, and morphometry," Nat. Methods 16, 67-70 (2019).

24. S. Laine, T. Karras, J. Lehtinen, and T. Aila, "High-Quality Self-Supervised Deep Image Denoising," in Advances in Neural Information Processing Systems (2019), pp. 6970-6980.

25. A. Krull, T.-O. Buchholz, and F. Jug, "Noise2Void-Learning Denoising from Single Noisy Images," in Proceedings of the IEEE Conference on Computer Vision and Pattern Recognition (2019), pp. 2129-2137.

26. K. Diederik and J. L. Ba, "ADAM: A Method for Stochastic Optimization," in AIP Conference Proceedings (2014), Vol. 1631, pp. 58-62.

27. K. Dabov, A. Foi, V. Katkovnik, and K. Egiazarian, "Image denoising with block-matching and 3D filtering," in Image Processing: Algorithms and Systems, Neural Networks, and Machine 
bioRxiv preprint doi: https://doi.org/10.1101/2020.06.17.158097; this version posted June 18, 2020. The copyright holder for this preprint (which was not certified by peer review) is the author/funder, who has granted bioRxiv a license to display the preprint in perpetuity. It is made available under aCC-BY-NC-ND 4.0 International license.

Learning (SPIE, 2006), Vol. 6064, p. 606414.

28. Z. Wang, A. C. Bovik, H. R. Sheikh, and E. P. Simoncelli, "Image quality assessment: From error visibility to structural similarity," IEEE Trans. Image Process. 13, 600-612 (2004). 